\title{
Unanticipated intra-operative finding of pulmonary artery tumour thromboembolism during radical nephrectomy and caval thrombectomy: Case report and management
}

\author{
Rajan Sharda, MD; Raymond Deutscher, MD; ; Chris Christodoulou, MD,; David Horne, MD; Darren H. Freed, \\ MD, FRCSC; ${ }^{+\dagger}$ Thomas McGregor, MD, FRCSC*
}

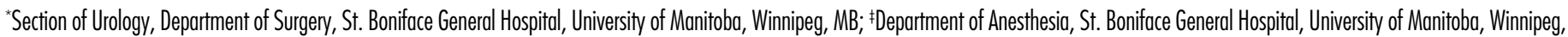
MB; ‘Section of Cardiac Surgery, Department of Surgery, St. Boniface General Hospital, University of Manitoba, Winnipeg, MB

Cite as: Can Urol Assoc J 2013;7(5-6):e381-5. http://dx.doi.org/10.5489/cuaj.1224 Published online May 13, 2013.

\section{Abstract}

We report a case of an unanticipated intra-operative transesophageal echocardiography (TEE) finding of pulmonary artery thromboembolism in a 72-year-old woman being prepared for radical nephrectomy and caval thrombectomy. Upon intra-operative TEE to evaluate the extent of caval thrombus, we found a pulmonary artery tumour thromboembolism in an otherwise asymptomatic patient after induction and prior to surgery. A chest computed tomography confirmed a large saddle tumour thromboembolus. A multidisciplinary approach was used to facilitate radical nephrectomy with caval thrombectomy and pulmonary artery thromboembolectomy. This case shows the importance of adequate perioperative imaging and use of intra-operative TEE to evaluate the extent of disease. To our knowledge, we are the first to present a case of RCC with cava tumour thrombus in which the pulmonary artery tumour thromboembolism was detected incidentally on intraoperative TEE.

\section{Introduction}

Renal cell carcinoma (RCC) with associated vena cava tumour thrombus represents $4 \%$ to $10 \%$ of RCC cases, with $1 \%$ of cases having tumour thrombus involving the right atrium. ${ }^{1}$ Concomitant renal neoplasm with pulmonary tumour-thromboembolism is even more uncommon and carries a poor prognosis. ${ }^{2}$ Most cases arise from preoperative findings on chest imaging (for a symptomatic patient) or from intraoperative embolization during caval manipulation leading to emergency chest surgery to remove the tumour embolus. ${ }^{3,4}$ We report a case of a renal neoplasm with caval tumour thrombus with an unanticipated finding of pulmonary tumour thromboembolism found on routine intraoperative transesophageal ultrasound.

\section{Case report}

A 72-year-old female lifelong smoker with a history of hypertension and hyperlipidemia presented with gross hematuria while vacationing in the United States. A ureteric stent was inserted to relieve clot colic and facilitate transfer back to Canada. Cystoscopy and right retrograde pyelography were also performed to further workup the hematuria and both were negative. Follow-up imaging by a community urologist confirmed a right renal mass. A computed tomography (CT) of the abdomen with intravenous contrast revealed an enhancing 5-cm right renal mass with no obvious evidence of distant metastasis, local invasion or venous involvement (Fig. 1). The patient was referred to a tertiary centre for consideration of laparoscopic radical nephrectomy. The patient returned to the emergency room 2 weeks later with significant gross hematuria, stable hemoglobin and otherwise completely asymptomatic, and denying any cardiorespiratory symptoms. Repeat imaging again showed the same rightsided enhancing mass, but now also showed clear evidence of a level II caval thrombus residing just caudal to the liver (Fig. 2). A magnetic resonance image better defined the full extent of vena cava tumour thrombus (Fig. 3). The patient was admitted to hospital for anesthesiology assessment and prepared for a radical nephrectomy and caval thrombectomy to be performed on an urgent basis.

Following a general anesthetic and surgical draping, routine transesophageal echocardiography (TEE) was performed to assess cardiac hemodynamics, as well as the cephalad extent of the tumour thrombus. According to the TEE examination as per Society of Cardiovascular Anesthesiologists/ American Society of Echocardiography guidelines, we incidentally found a tumour thrombus clearly identified within the right pulmonary artery (Fig. 4).

The patient was then transferred to the radiology department while still under general anesthetic for emergent CT 


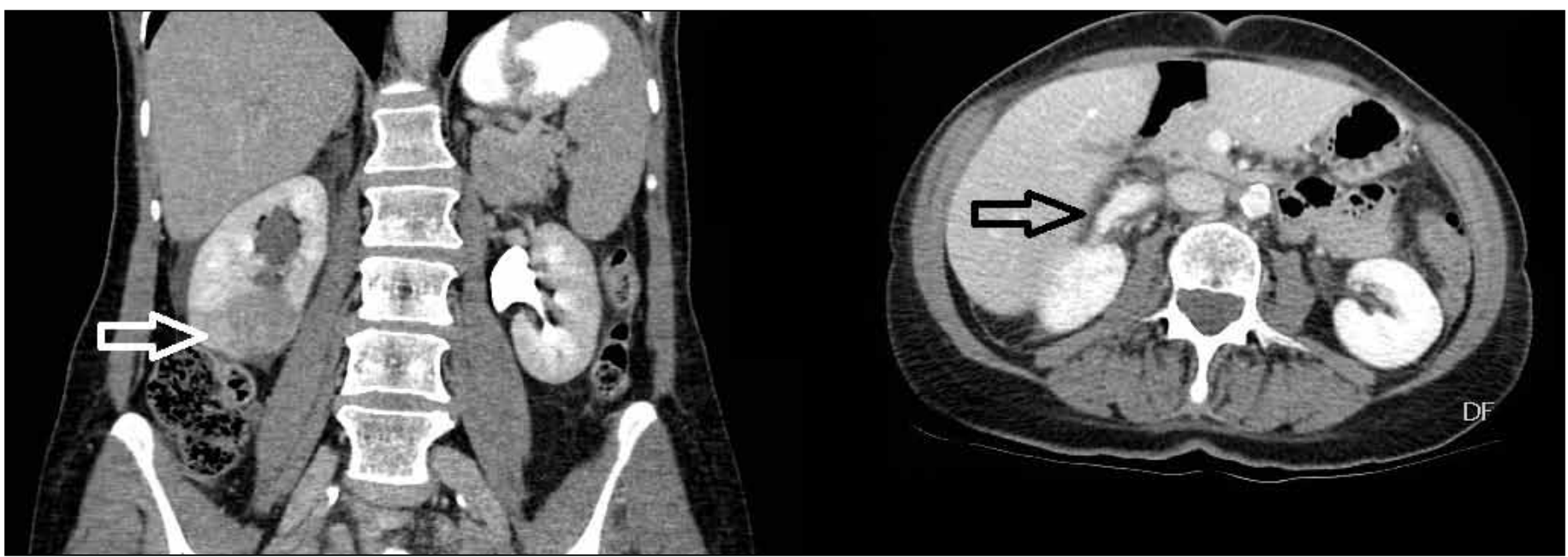

Fig. 1. A computed tomography scan at initial presentation. On the left, delayed phase coronal imaging showing an approximate 5-cm right lower pole renal mass. On the right, axial image of renal hilum showing no evidence of renal vein tumour thrombus and absence of any hilar nodes.

chest to better define the anatomy and extent of pulmonary tumour thromboembolus. As suspected, the CT showed a large saddle embolus with characteristics consistent with tumour thromboembolus (Fig. 5).

Given that the patient was hemodynamically stable and showed no cardiac or pulmonary compromise, a right radical nephrectomy and caval thrombectomy was performed via a chevron incision. This was followed by a sternotomy and pulmonary artery thromboembolectomy with cardiopulmonary bypass. To facilitate removal of the tumour, circulatory arrest was employed for 8 minutes at $20^{\circ} \mathrm{C}$ core body temperature. This gave excellent visualization of the

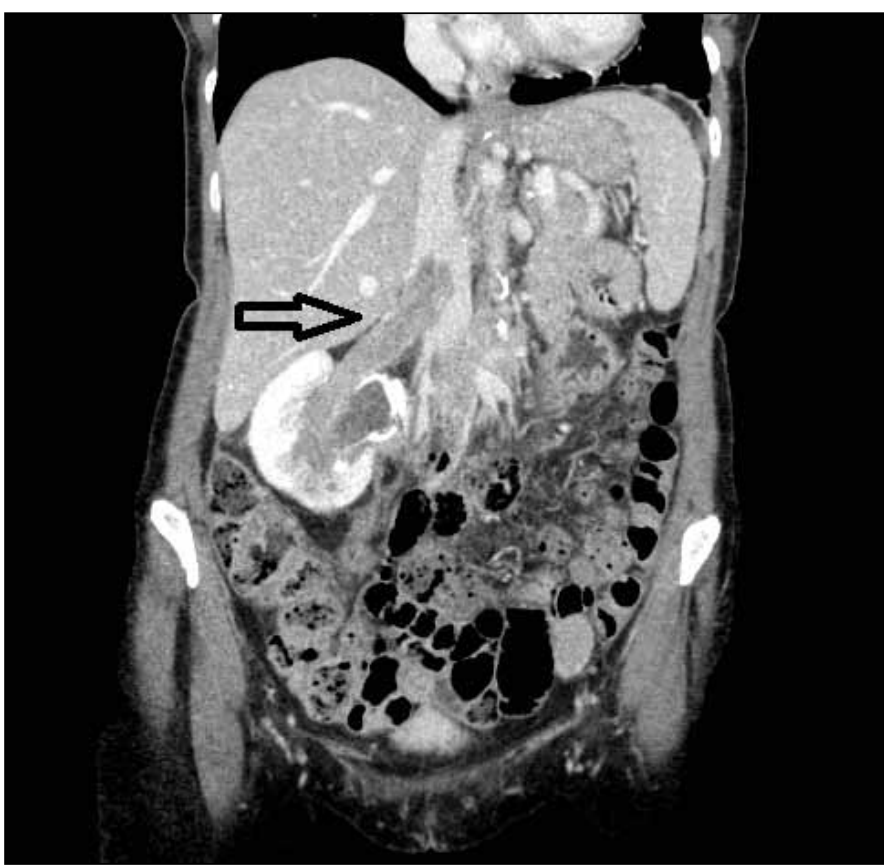

Fig. 2. A computed tomography scan showing right renal mass with arrow pointing to tumour thrombus extending into vena cava (level II caval thrombus). origins of the subsegmental arteries, and allowed removal of all visible thrombus with the exception of the left lower lobe and lingual. Because the tumour was extremely friable, and yet adherent to the arterial wall, it was not possible to remove all the tumour thrombus in this region. Gross and microscopic pathological specimens confirmed the presence of RCC caval tumour thrombus with thromboembolized tumour within the pulmonary arteries (Fig. 6, Fig. 7).

The patient hospital stay was complicated by new diagnosis of paroxysmal atrial fibrillation. Cardiology consultation was acquired and the patient was medically converted with diltiazem and initiated on metoprolol and anticoagulation.

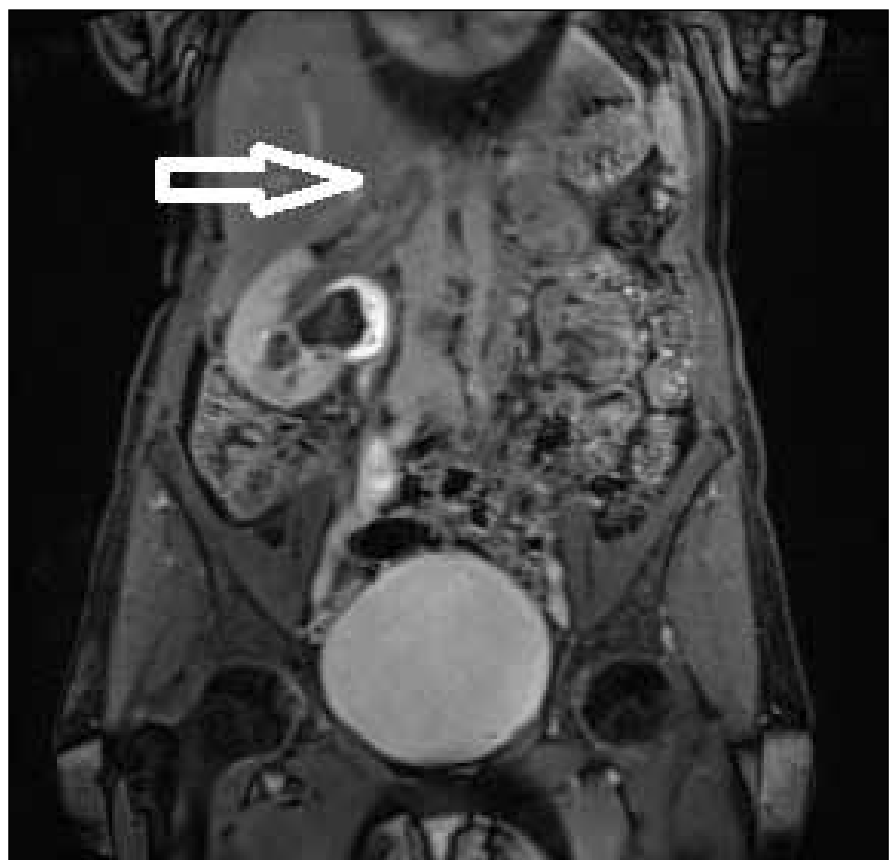

Fig. 3. A magnetic resonance image showing the extent of caval tumour thrombus extension. 


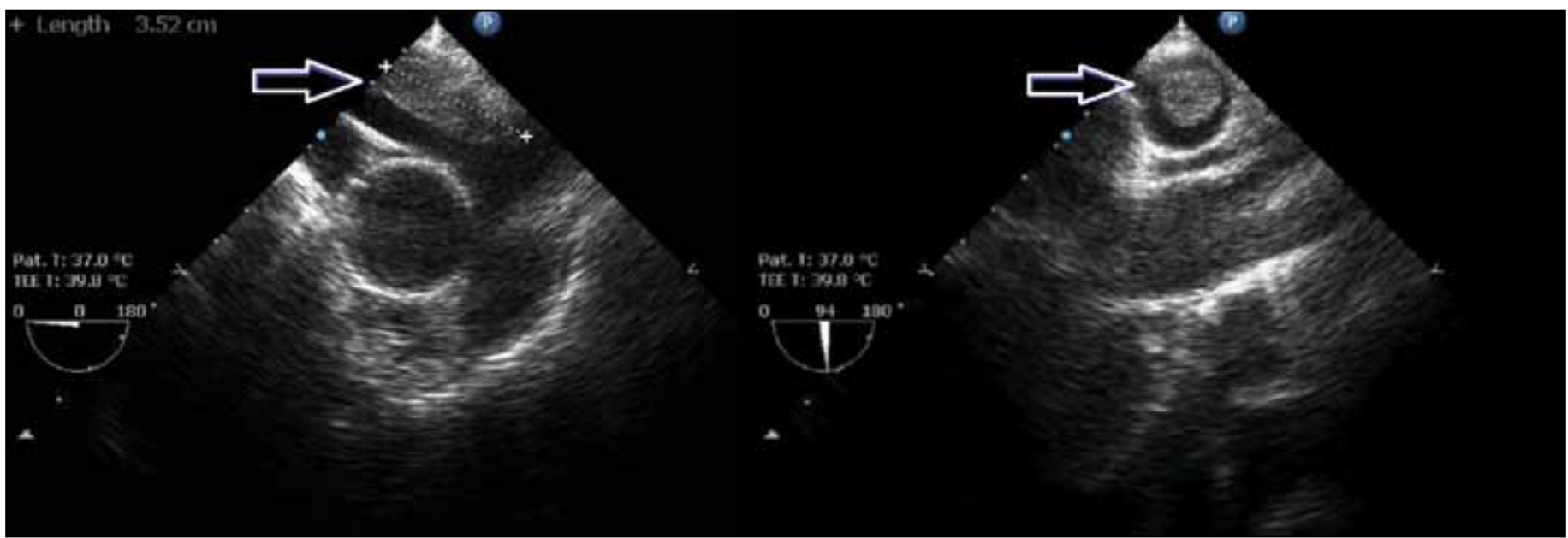

Fig. 4. A transesophageal echocardiography demonstrating pulmonary artery tumour embolus involving right pulmonary artery. On the left, coronal view showing tumour embolus in right pulmonary artery with partial extension into left pulmonary artery. On the right, sagittal view showing the extent of tumour embolus involving the lumen of the pulmonary artery.

The patient was discharged from hospital on postoperative day 12 with no significant perioperative complications. Consultation with medical oncology was then arranged for consideration of tyrosine kinase inhibitor therapy. Repeat imaging at 1 month showed no evidence of residual tumour or recurrence within the abdomen. However, a small thrombus remnant remained within the segemental pulmonary artery, which could not be differentiated between bland or tumour thrombus. Pathology confirmed pT3c disease and showed Fuhrman grade 4 clear cell RCC with $<30 \%$ sarcomatoid differentiation. Pulmonary artery embolectomy specimen was consistent with tumour emboli and showed the same histology as the renal specimen (Fig. 8).

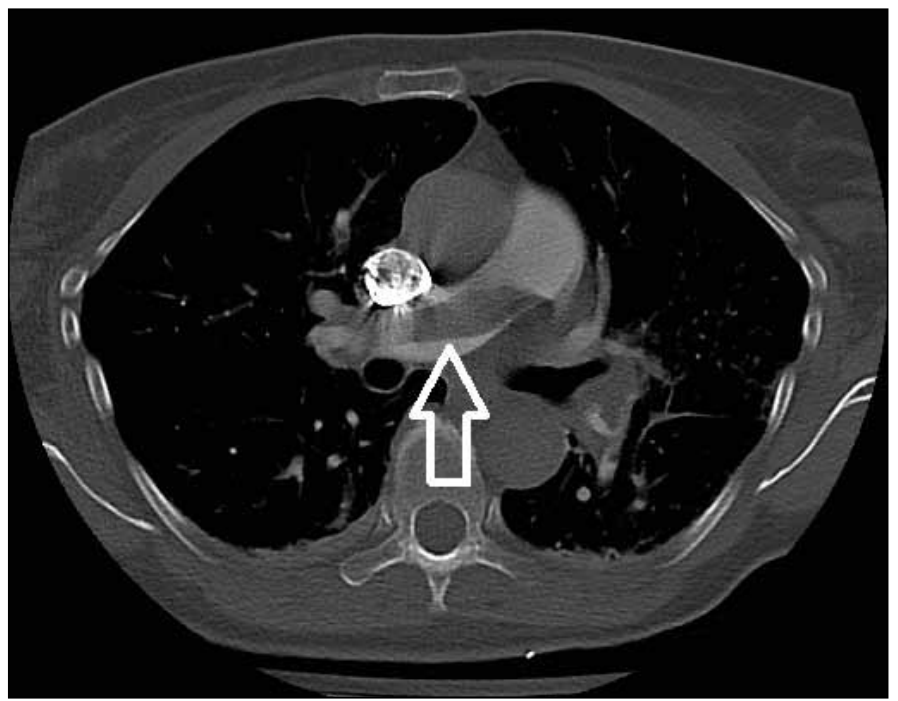

Fig. 5. A computed tomography scan of the chest showing extent of tumour saddle embolus. Tumour embolus extents into both main pulmonary arteries and segmental branches bilaterally.

\section{Discussion}

RCC with associated caval tumour thrombus presents a significant surgical challenge that requires proper preoperative workup and surgical planning to treat successfully. Importantly, to determine the extent and level of the tumour thrombus one or more imaging studies must be ordered preoperatively. Most cases present with $\mathrm{CT}$ as the initial imaging modality, which often identify or can suggest the

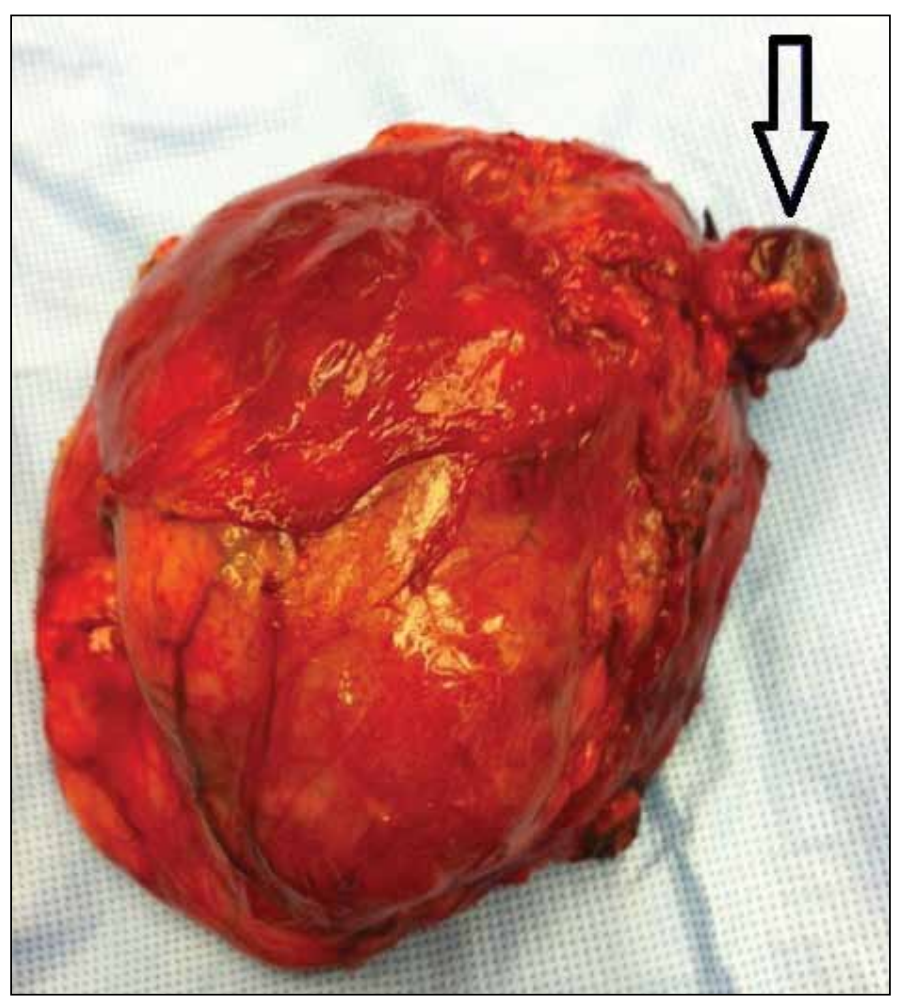

Fig. 6. Gross surgical specimen of right kidney and caval tumour thrombus. 


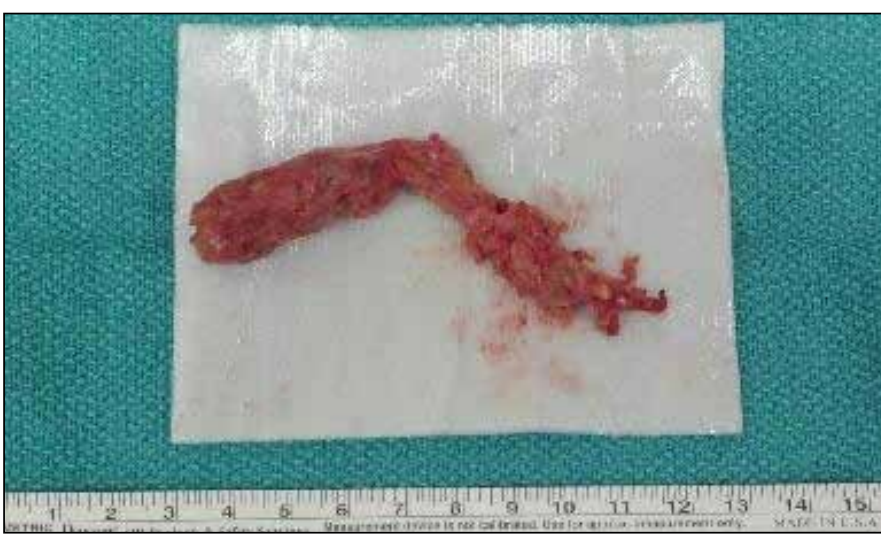

Fig. 7. Gross surgical specimen of excised pulmonary artery saddle tumour embolus.

presence of tumour thrombus. However, MRI is the preferred imaging modality as it reliably defines both the extent of a tumour thrombus and also distinguishes between tumour and bland thrombus. ${ }^{5}$ Other less frequently used preoperative imaging modalities include venacavography, Doppler ultrasonograpy and TEE.

Intraoperatively, TEE has become routine to determine the cephalad extent of the tumour thrombus. Firstly, this is important to rule out thrombus growth during the interval between the preoperative imaging and the operating room. Secondly, TEE is able to assess and help monitor cardiac hemodynamics during caval mobilizing and clamping. Finally, TEE is able to detect distal pieces of tumour thrombus that may infrequently break loose and migrate into one of the cardiac chambers. ${ }^{6}$

In this case, TEE clearly showed the cephalad extent of the tumour thrombus (infrahepatic), but also incidentally identified a large floating mass within one of the pulmonary arteries. The pulmonary arteries are routinely assessed when following the protocol for TEE. This was fortunate for the patient, who at the time was preoperatively asymptomatic and hemodynamically stable intraoperatively.

The literature shows that tumour thromboembolism into the pulmonary artery is rare for RCC and usually occurs during cava manipulation and mobilization, ${ }^{4,7,8}$ or is detected preoperatively when a patient has pulmonary symptoms and chest imaging reveals the finding. ${ }^{9}$ To our knowledge, we are the first to present a case of RCC with cava tumour thrombus in which the pulmonary artery tumour thromboembolism was detected in such a manner, incidentally on TEE. By further defining the size and extent of the pulmonary component with chest $\mathrm{CT}$, we were able to safely and effectively manage both the retroperitoneal component (kidney and vena cava) and pulmonary component of the disease.

\section{Conclusion}

RCC with vena cava tumour thrombus and pulmonary artery tumour thromboembolism presents a unique and challenging case for the surgical team. This case demonstrates the importance of intraoperative TEE during resection of RCC with tumour thrombus. Furthermore, this highlights the importance of a multidisciplinary approach between the urologist and anesthesiologist in effectively treating this disease, with involvement of the cardiac team when tumour thrombus either extends into the chest or embolizes further as in this case.

\section{Competing interests: None declared.}

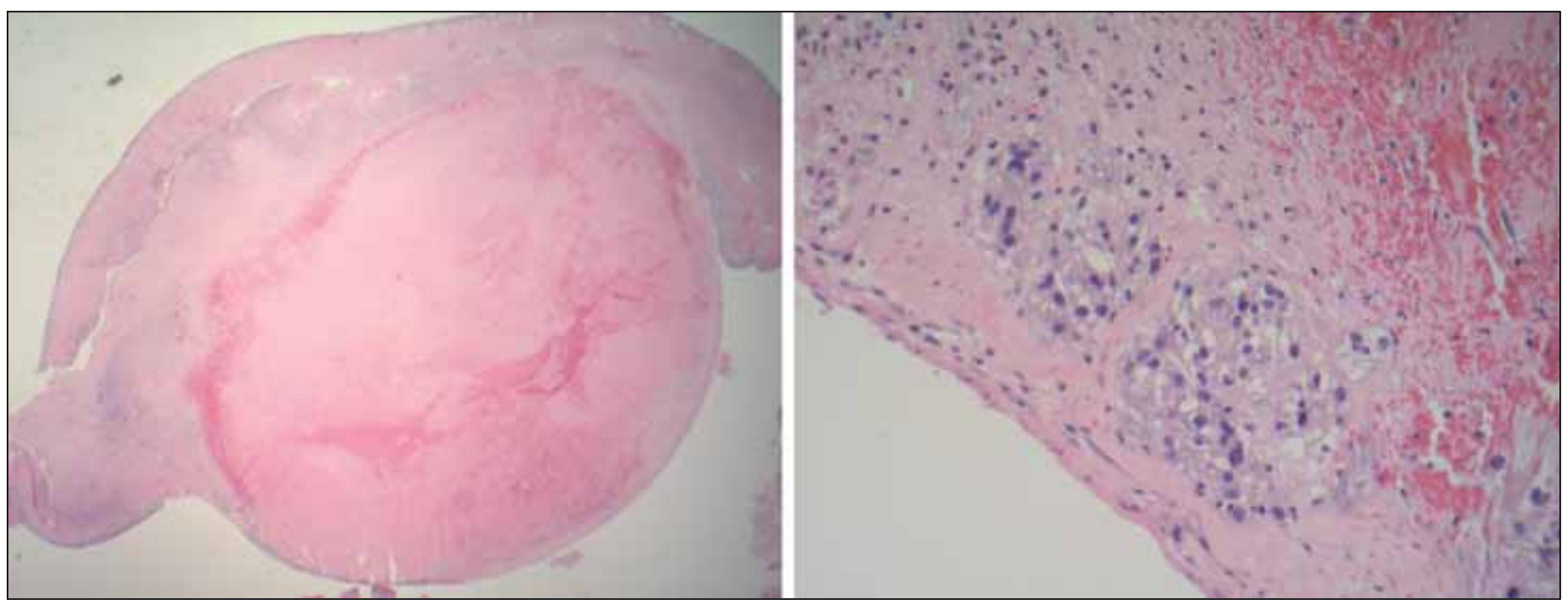

Fig. 8. On the left, low power magnification of thrombus showing cast-like shape containing a clear cell tumour. On the right, high power view shows large cells with cleared cytoplasm consistent with renal cell carcinoma. 
This paper has been peer-reviewed.

\section{References}

1. Marshall F, Dietrick D, Baumgartner W, et al. Surgical management of renal cell carcinoma with intracaval neoplastic extension above the hepatic veins. J Urol 1988;139:1166-72.

2. Bassiri AG, Haghigi B, Doyle RL, et al. Pulmonary tumor embolism. Am J Respir Crit Care Med 1997;155:2089-95. http://dx.doi.org/10.1164/airccm.155.6.9196119

3. Nagasaka $S$, Taniguchi $S$, Kobayashi $S$, et al. Successful treatment of intraoperative pulmonary embolism from renal cell carcinoma. Heart Vessels 1997;12:199-202. http://dx.doi.org/10.1007/BF02767048

4. Tsubo $\mathrm{T}$, Ebina $\mathrm{M}, 0$ tomo $\mathrm{N}$, et al. Accurate detection of pulmonary embolism using epicardial echocardiography during right nephrectomy in a patient with renal cell carcinoma. J Cardiothorac Vasc Anesth 1998;12:684-5. http://dx.doi.org/10.1016/S1053-0770(98)90244-0

5. Heidenreich A, Ravery V; European Society of Oncological Urology. Preoperative imaging in renal cell cancer. World J Urol 2004;22:307-15. http://dx.doi.org/10.1007/s00345-004-0411-2
6. Sigman DB, Hasnain JU, Del Pizzo JJ, et al. Realtime transesophageal echocardiography for intraoperative surveillance of patients with renal cell carcinoma and vena caval extension undergoing radical nephrectomy. J Urol 1999;161:36-8. http://dx.doi.org/10.1016/S0022-5347(01)62054-8

7. Sasaoka N, Kawaguchi M, Sha K, et al. Intraoperative immediate diagnosis of acute obstruction of tricuspid valve and pulmonary embolism due to renal cell carcinoma with transesophageal echocardiography. Anesthesiology 1997;87:998-1001. http://dx.doi.org/10.1097/00000542-199710000-00041

8. Kobayashi T, Ogura K, Nishizawa K, et al. Successful recovery from a massive pulmonary arter tumor embolism occurring during surgery for renal cell carcinoma. Int I Urol 2004;11:114-6. http://dx.doi. org/10.1111/j.1442-2042.2004.00743.x

9. Kayalar N, Leibovich B, Orszulak T, et al. Concomitant surgery for renal neoplasm with pulmonary tumor embolism. J Thorac Cardiovasc Surg 2010;139:320-5. http://dx.doi.org/10.1016/i.jtcus.2009.04.021

Correspondence: Dr. Tom McGregor, St. Boniface General Hospital, Department of Surgery, Z3032409 Tache Ave., Winnipeg, MB R2H 2A6; tmcgregor2@sbgh.mb.ca 